\title{
Improvement of dietary quality with the aid of a low glycemic index diet in Asian patients with type 2 diabetes mellitus.
}

\begin{abstract}
This randomized controlled study was conducted to determine the effect of low glycemic index (GI) dietary advice on eating patterns and dietary quality in Asian patients with type 2 diabetes $(\mathrm{T} 2 \mathrm{DM})$. Asian patients with T2DM $(\mathrm{N}=104)$ were randomized into 2 groups that received either low GI or conventional carbohydrate exchange (CCE) dietary advice for 12 weeks. Nutritional prescriptions were based on the medical nutrition therapy for T2DM, with the difference being in the GI component of the carbohydrates. Dietary intake and food choices were assessed with the use of a 3-day food record. At week 12, both groups achieved the recommendations for carbohydrate (52 $\pm 4 \%$ and $54 \pm 4 \%$ of energy) and fat (30 $\pm 4 \%$ and $28 \pm 5 \%$ of energy) intake. There were no significant differences in the reported macronutrient intake in both groups. With the low GI diet, crude fiber and dietary calcium intake increased, while the dietary GI reduced. Subjects in the lowest dietary glycemic index/glycemic load (GI/GL) quartile consumed more parboiled/basmati rice, pasta, milk/dairy products, fruits, and dough, which are foods from the low GI category. There was a significant reduction in the hemoglobin $\mathrm{A}(1 \mathrm{c})$ level at week 12 for patients in the lowest GI/GL quartile $(\Delta=-0.7 \pm 0.1 \%)$ compared with those in the highest GI/GL quartile $(\Delta=-$ $0.1 \pm 0.2 \%$ ). These results demonstrate the ability of low GI dietary advice to improve the dietary quality of Asian patients with T2DM.
\end{abstract}

Keyword: Dietary; Low glycemic index; Type 2 diabetes. 